\section{Entrevista al Dr. Eugenio Beltrán-Aguilar, Director Asociado del Centro Colaborador de la Organización Mundial de la Salud (OMS) en la Universidad de Nueva York}

\author{
Interview to Dr. Eugenio Beltrán- \\ Aguilar, Associate Director World Health \\ Organization Collaborating Center at New \\ York University
}

\section{Entrevista}

\author{
Ana Arana Sunohara ${ }^{1, a}$ \\ 1 Universidad Peruana Cayetano Heredia, Facultad de \\ Estomatología, Lima, Perú. \\ ${ }^{a}$ Master of Science in Dental Public Health. \\ Correspondencia: \\ Ana Arana Sunohara: ana.arana@upch.pe \\ Universidad Peruana Cayetano Heredia, Facultad de \\ Estomatología. Av. Honorio Delgado 430, San Martín de \\ Porres \\ ORCID: 0000-0003-0267-1580
}

Conflicto de intereses: la autora declara no tener conflictos de interés.

\section{Fuente de financiamiento: ninguno}

Recibido: $19 / 07 / 20$

Aceptado: 20/07/20

Publicado: 04/08/20

\begin{abstract}
Resumen
Eugenio Beltrán-Aguilar comparte en esta entrevista su visión de lo avanzado en América Latina y los desafíos pendientes en materia de salud pública, investigación y desarrollo tecnológico en el contexto de la pandemia por COVID-19. Resalta la importancia de realizar estudios epidemiológicos e institucionalizar sistemas de vigilancia epidemiológica que generen información actualizada y de calidad que sirva para dar sustento a las políticas públicas ante problemas persistentes como la caries dental y los nuevos desafíos como el cáncer orofaríngeo asociado a infección por el virus del papiloma humano $(\mathrm{VPH})$. Analiza también el impacto de la pandemia en la enseńanza de la Odontología y la práctica odontológica, a la vez que señala rutas para avanzar en investigación y mejorar nuestro conocimiento sobre los posibles impactos de la pandemia en la población. Todo esto, en un contexto de debilitamiento de los organismos multilaterales de salud pública.
\end{abstract}

Palabras clave: Vigilancia epidemiológica; Salud global; Pandemias (fuente: DeCS BIREME).

\begin{abstract}
Eugenio Beltrán-Aguilar shares in this interview his vision of the advances and pending challenges in Latin America regarding public health, research and technological development in the context of the pandemic by COVID-19. It highlights the importance of carrying out epidemiological studies and institutionalizing epidemiological surveillance systems to generate up-to-date and quality information that serves to support public policies in the face of persistent problems such as dental caries and new challenges such as oropharyngeal cancer associated with human papillomavirus (HPV) infection. It also analyzes the impact of the pandemic on dental education and dental practice, while pointing out routes for advanced research and to improve our knowledge on the possible impacts of the pandemic on the population. All this, in a weakening context of multilateral public health organizations.
\end{abstract}

Keywords: Epidemiologic surveillance; Global health; Pandemics (source: MeSH NLM).

(c) Los autores. Este artículo es publicado por la revista Odontología Sanmarquina de la Facultad de Odontología, Universidad Nacional Mayor de San Marcos. Este es un artículo de acceso abierto, distribuido bajo los términos de la licencia Creative Commons Atribucion - No Comercia_Compartir Igual 4.0 Internacional. (http://creativecommons.org/licenses/by-nc-sa/4.0/) que permite el uso no comercial, distribución y reproducción en cualquier medio, siempre que la obra original sea debidamente citada. 


\section{Introducción}

Eugenio Beltrán-Aguilar es en la actualidad Profesor Adjunto del Departamento de Epidemiología y Promoción de la Salud de la Facultad de Odontología de la Universidad de Nueva York y Director Asociado de Epidemiología y Vigilancia para el Centro Colaborador de la Organización Mundial de la Salud (OMS). Odontólogo de formación, con especialidad en Epidemiología, el Dr. Beltrán-Aguilar tiene una reconocida trayectoria en la salud pública internacional, como funcionario del Centro de Control y Prevención de Enfermedades de los Estados Unidos y consultor de la Organización Panamericana de la Salud. Esta entrevista se realiza pasados más de cien días desde que se identificó el primer caso de COVID-19 en el Perú. En ella, desde una perspectiva de salud global, indagamos por su visión de lo avanzado en América Latina en materia de salud pública bucal y los desafíos que se deben afrontar para proteger los logros y seguir avanzando en la mejora de la salud bucal en el presente contexto de pandemia.

\section{Entrevista}

¿Cuál era el estado de la salud bucal en América Latina antes de la pandemia por COVID-19? ¿Cómo se compara frente a otras regiones del globo? ¿Ha habido algún progreso en los últimos 10 años?

Para entender el nivel de salud bucodental de una población se requiere datos e información frecuente, lo cual, con algunas excepciones, no han sido prioridades de los países o de las organizaciones internacionales en los últimos 10 años.

Existe un gran interés en hacer estudios, sobre todo epidemiológicos, y con rigor científico gracias a colaboraciones inter-institucionales. Desafortunadamente, publicados o no, la gran mayoría de estos estudios no son utilizados en el desarrollo de políticas o intervenciones. Las políticas de salud bucodental en el mundo, con algunas excepciones, están condicionadas al entorno político local. Ministerios o Secretarias de Salud con un alto compromiso para la salud bucal, en forma de departamentos o unidades especializadas, logran tener más alto impacto poblacional a través de medidas preventivas.

En los noventa, cuando se produjo información epidemiológica en varios países de la región, se reportó una disminución general en la prevalencia y severidad de caries dental. Esta información está desactualizada, porque la mayoría de los países de la región y sus unidades administrativas no mantienen sistemas de vigilancia epidemiológica. Tampoco hay información sobre otras enfermedades o condiciones excepto fluorosis en aquellos países que iniciaron y fomentaron la fluoruración del agua, la sal o leche.

Algunos países como Brasil, Costa Rica y Chile mantienen un sistema de vigilancia epidemiológica de cobertura poblacional. Otros países, como es el caso del Perú, han hecho más de un estudio epidemiológico, pero no hay información actualizada sobre la vigencia de los pro- gramas de fluoruración, y sobre el monitoreo biológico y químico que recomendó la OPS.

Existe información sobre un incremento del cáncer orofaríngeo asociado a infección por el virus del papiloma humano (VPH). El 2018 se realizó una reunión internacional en Washington DC donde se discutió la evidencia microbiológica, epidemiológica y preventiva. Los niveles de vacunación de adolescentes en América Latina no son altos. Es posible que las campañas de vacunación contra VPH tengan un efecto sobre esto tipo de cáncer, pero su efecto requerirá estudios a largo plazo.

Hay países en la región con variados intereses en la investigación epidemiológica y de salud bucal en sus poblaciones como México, Brasil y Argentina.

Los países del África tienen un programa regional de salud bucal. Algunos países del Sudeste Asiático están ingresando en la etapa creciente de la caries dental debido al reemplazo de la dieta tradicional por la occidental (Vietnam, Cambodia y Laos). Otros, como Tailandia, tienen programas nacionales preventivos a base de barnices en las escuelas. Ciudades como Hong Kong, Singapur y Ho Chi Minh, tienen programas de fluoruración del agua por más de 30 años que han mostrado una disminución de la prevalencia de caries dental. En el subcontinente indio, Irán y China, existe fluorosis endémica producida no solo por exceso de fluoruro en el agua, sino por el uso de carbón y exposiciones ambientales. En estos países hay reticencia a la utilización de fluoruros como medida preventiva. Además en el subcontinente indio, se reporta la tasa de cáncer oral y faríngeo más alta del mundo debido al consumo de tabaco en diferentes formas.

Los países del oeste europeo (y en menor grado los del este), Canadá, EEUU, Australia y Nueva Zelandia, han mostrado avances en la prevención de la caries dental producido por la utilización de fluoruros en forma sistémica, tópica y profesional. A eso se suma la disponibilidad de servicios dentales para la mayoría de sus poblaciones. Los temas vigentes en estos países, sobre todo en Europa, EEUU y Canadá son las desigualdades en grupos poblacionales con diferentes niveles de desarrollo social, comunidades nativas, minorías e inmigrantes. En general los países con más homogeneidad social y desarrollo económico tienen los niveles más altos de salud bucodental.

¿Puede mencionar algunos casos de éxito en la región (América Latina)? ¿Qué paises y qué politicas públicas, estrategias, enfoques, intervenciones han impulsado para mejorar la salud bucal de sus ciudadanos de manera exitosa? ¿Puede mencionar experiencias de éxito en otros paises que puedan ser de utilidad para la región?

Los programas de fluoruración de la sal y del agua han documentado una disminución en la prevalencia de caries dental y un aumento controlado en la fluorosis dental en Costa Rica, Jamaica, México, Colombia, Ecuador, Chile, Argentina, Uruguay y Brasil. Pero no existe información reciente. Los programas requieren 
un componente administrativo y un monitoreo biológico y químico, además de la colaboración de la industria salinera y de los operadores de los sistemas de agua potable. Tal nivel de coordinación requiere de equipos de trabajo bajo el liderazgo de la entidad nacional o regional de salud.

En Chile existe un programa activo de fluoruración de la leche en regiones administrativas donde no hay fluoruración del agua.

En Argentina, la Región del Gran Buenos Aires tiene fluoruración del agua. Además tiene varios proyectos preventivos con sellantes, SDF, y barnices que son manejados por la Universidad de Buenos Aires, aprovechando el acceso a intervenciones preventivas de todos los escolares en la Región del Gran Buenos Aires. En Mendoza se ha reportado fluorosis dental.

Brasil tiene muchos programas similares gracias al apoyo financiero de los estados y de las instituciones académicas. Previo a la actual administración había financiamiento central para hacer investigación que incluía dinero para presentar sus trabajos en reuniones internacionales.

Muchos de estos programas a nivel regional y nacional tienen un tiempo de vida limitado o sufren problemas de financiación en etapas de crisis económica. Desafortunadamente estos programas no incluyen una evaluación de costo-beneficio. El principal problema es que la mayoría de estas intervenciones no son difundidas apropiadamente.

En el contexto de la pandemia por el COVID-19 ¿Cuáles son los impactos que tendrá la pandemia en la salud bucal de América Latina? ¿Cuáles son las recomendaciones de política pública que la OPS/OMS hace a los países de América Latina en este contexto?

La pandemia obligó a varios países a restringir el servicio médico ambulatorio donde están incluidos los servicios odontológicos, salvo casos de emergencia (identificados como dolor, infección y complicaciones posteriores por falta de atención). La razón principal detrás de esta medida fue disminuir la posibilidad de contagio y reducir el uso de equipo de protección personal que podría utilizarse en pacientes que están infectados por SARSCoV-2.

En la actualidad, en los EEUU, se puede administrar tratamiento dental más allá de emergencias. En algunos estados se habla de retornar a la normalidad, pero incluyendo medidas adicionales de protección a nivel de la consulta dental debido a que el SARS-CoV-2 se transmite fácilmente por aerosoles. Las precauciones adicionales implican un coste adicional que será pasado al paciente o a su seguro. Los programas de ayuda nacional como Medicaid se verán afectados porque los niveles inferiores de reembolso determinará que algunos proveedores no acepten a este tipo de pacientes por las condiciones económicas luego de 3-4 meses sin ingreso económico, además de la necesidad de hacer cambios en los ambientes clínicos y administrativos de la consulta.
No existe recomendación de la OPS sobre la reapertura de servicios dentales en la región. Hasta julio, 2020, no existe información sobre el estado de atención odontológica en los países de la región porque la OPS no mantiene un sistema de vigilancia. Es posible que las normativas nacionales y locales de reabrir la consulta odontológica sigan las recomendaciones globales de reducir la transmisión y mantener un sistema que puede atender cualquier reemergencia con identificación, rastreo y seguimiento.

Hay algunas hipótesis sobre el efecto de la pandemia en la salud bucodental. Pero no habría que esperar el retorno a una normalidad para comenzar a evaluar dichos efectos. Las hipótesis varían dependiendo del estado de salud bucodental y el tipo de servicios recibidos antes de la pandemia.

En la situación "óptima", esto es una población que recibe tratamiento preventivo y curativo frecuente, el efecto podría ser mínimo o un aumento en la incidencia de caries dental, sobre todo en niños; además probablemente haya un aumento en la gingivitis en mujeres embarazadas. Así mismo, es probable que algunos tratamientos hayan fallado y al no haber acceso a la atención, las personas necesiten un tratamiento más agresivo al levantarse las restricciones.

En la situación de más desventaja, aquellas poblaciones que no reciben tratamiento dental de rutina, se reportarán estados de enfermedad más avanzada con dolor, infección y afección en las condiciones de vida y que probablemente lleguen a niveles extremos incluyendo la pérdida dentaria con la secuela clínica y social que conlleva.

Es probable, que la población en general reporte episodios frecuentes de dolor, infección y necesidad de tratamiento quimioterapéutico.

Las facultades y escuelas de Odontología se enfrentan a una situación jamás pensada: la necesidad de hacer cambios en la forma cómo se enseña los aspectos teóricos y clínicos. No existen recomendaciones universales al respecto y en los EEUU, y sus más de 60 facultades están generando sus propios planes. Hasta mediados de junio, algunas facultades estaban poniendo en prueba dichos programas comenzando por prestación de servicios de emergencia dental por profesores o alumnos graduados. La mayoría de facultades tienen planeado reabrir todos los servicios a mediados de agosto cuando se inicia el año académico. La mayoría de las clases teorías serán por internet. Esto implica un grave efecto económico en las facultades porque una proporción alta del presupuesto es cubierto por servicios prestados a la población.

La OPS y la OMS tienen recomendaciones sanitarias para el COVID-19 pero no son específicas para salud bucodental. Por otro lado, el Centro para el Control y Prevención de Enfermedades (CDC) y otras instituciones gubernamentales de los EEUU, la Asociación Dental Americana y las organizaciones de las especialidades han publicado recomendaciones específicas para sus miembros. 
¿Qué prioridades de investigación son las recomendables? ¿Hacia dónde dirigimos la innovación y el desarrollo de nuevas tecnologias?

La investigación en salud bucal en América Latina se puede agrupar en cuatro categorías (por orden de frecuencia con algunas variaciones entre países):

a. Reportes de caso, intervenciones clínicas o revisiones de la literatura.

b. Evaluación de laboratorio o clínica de materiales dentales.

c. Estudios epidemiológicos en muestras poblacionales.

d. Estudios de ciencia básica.

La prioridad regional en investigación debería optar por estudios epidemiológicos (c) pero con aplicación de los resultados en el diseño de intervenciones preventivas y curativas. Así mismo (a) y (b) deben ser incorporadas dentro de la evidencia incremental. Una tendencia que felizmente se está superando es a repetir estudios cuando ya existe evidencia suficiente. Esto podría pensarse como contradictorio al principio que la ciencia se basa en la repetición. El argumento contrario es que en ambiente de financiación limitada, debe haber una prioridad en las agendas de investigación. Finalmente, existe muy pocos estudios evaluativos o de costo-beneficio.

En términos generales la innovación facilita la disponibilidad de evidencia, políticas y evaluación de intervenciones que, en conjunto, llevan a una mejoría de los niveles de salud. Desgraciadamente, muchas veces la innovación se entiende solo al aspecto tecnológico, por ejemplo, equipo o materiales, porque generalmente son las que reciben financiación de la industria.

¿Cuál es el estado actual del Programa Regional de Salud Bucal de la OMS? ¿Qué oportunidades de asistencia técnica o de colaboración existen en la región?

Desde diciembre del 2019 la Organización Panamericana de la Salud - OPS, oficina regional de la Organización Mundial de la Salud entró en una etapa crítica presupuestal debido a la negativa del gobierno de EEUU de pagar su contribución con el organismo regional. Esto es un reflejo de la política del actual gobierno norteamericano, no la de los funcionarios de la Secretaría (Ministerio) de Salud. Previamente el gobierno americano ha tratado de ejercer más injerencia en las decisiones administrativas de la OPS, por ejemplo en la coordinación del programa de vacunación.

El Programa Regional de Salud Bucal, que tuvo su pico en los años 1980s, fue reorganizado bajo diferentes estructuras administrativas y su presupuesto reducido a un mínimo. Los proyectos de fluoruración y de prácticas restaurativas atraumáticas (PRAT) que coordinó el Programa fueron financiados externamente por fundaciones filantrópicas, por organismos regionales como el Banco Interamericano de Desarrollo (BID) o, en el caso de la iniciativa regional Comunidades Libres de Caries, Salud Oral, Factores de Riesgo (SoFAR), por la industria privada. Mientras hubo dinero, se mantuvieron los programas regionales y el apoyo a los países. Por ejemplo, se organizaron varias reuniones sobre el tema de fluoruración, el control de infecciones en Odontología y la implementación del ART. En algún momento hubo apoyo al programa de poblaciones libres de caries dental y la rehabilitación de la profesión odontológica en Haití luego del terremoto, pero siempre con dinero de otras instituciones.

La agenda de trabajo de la OPS para el quinquenio 2014-2019 eliminó por completo el Programa Regional y mantiene a una colega como coordinadora con menos del 5\% de dedicación bajo el programa de curso de vida. El Programa Regional logró hace 4 años aprobar un Centro Colaborador en la Universidad de Nueva York, el cual ha apoyado algunos pedidos de asistencia con dinero de los respectivos países. El Centro Colaborador entra en un proceso de reevaluación a fines de año y es probable que pida traslado de gestión administrativa directa a la OMS, dependiendo de la decisión política de la administración.

La única solución para revertir el estatus actual organizativo en defensa de un nuevo programa regional es el pedido directo de los países miembros. La Asamblea General de Ministros de Salud debe pedir formalmente al Comité Ejecutivo que incorpore salud bucodental en el próximo ciclo presupuestal e identifique una persona dentro de la organización como Consejero (Asesor) para que desarrolle un plan de acción entro del plan estratégico de la OPS.

Desgraciadamente OPS, como ocurre con muchas instituciones internacionales y ONGs están constantemente migrando en la dicotomía de la integración vertical (que identifica los problemas) y la integración horizontal (que convierte en anónimas a las necesidades). Como la salud bucodental es vista como un componente de menor cuantía por las autoridades sanitarias en OPS y en los países, no llega a los niveles de decisión si es que la profesión y los profesionales de salud bucodental no hacen su parte para demostrar que, como dijo el Cirujano General de los EEUU (un médico), sin salud bucodental no hay salud en general.

\section{Comentarios finales}

La integridad de la profesión depende de la calidad, preparación y compromiso de sus miembros, sus elegidos para dirigir las instituciones académicas y gremiales y de su integración con otras profesiones. Todos estos eslabones deberían estar coordinados permitiendo la utilización de las mejores intervenciones poblaciones e individuales en el control de las enfermedades bucodentales. Más aún, como consecuencia directa del cambio generacional constante debe haber una constante evaluación de la misión que la sociedad ha puesto en la profesión. 
Entrevista al Dr. Eugenio Beltrán-Aguilar, Director Asociado del Centro Colaborador de la Organización Mundial de la Salud (OMS)...

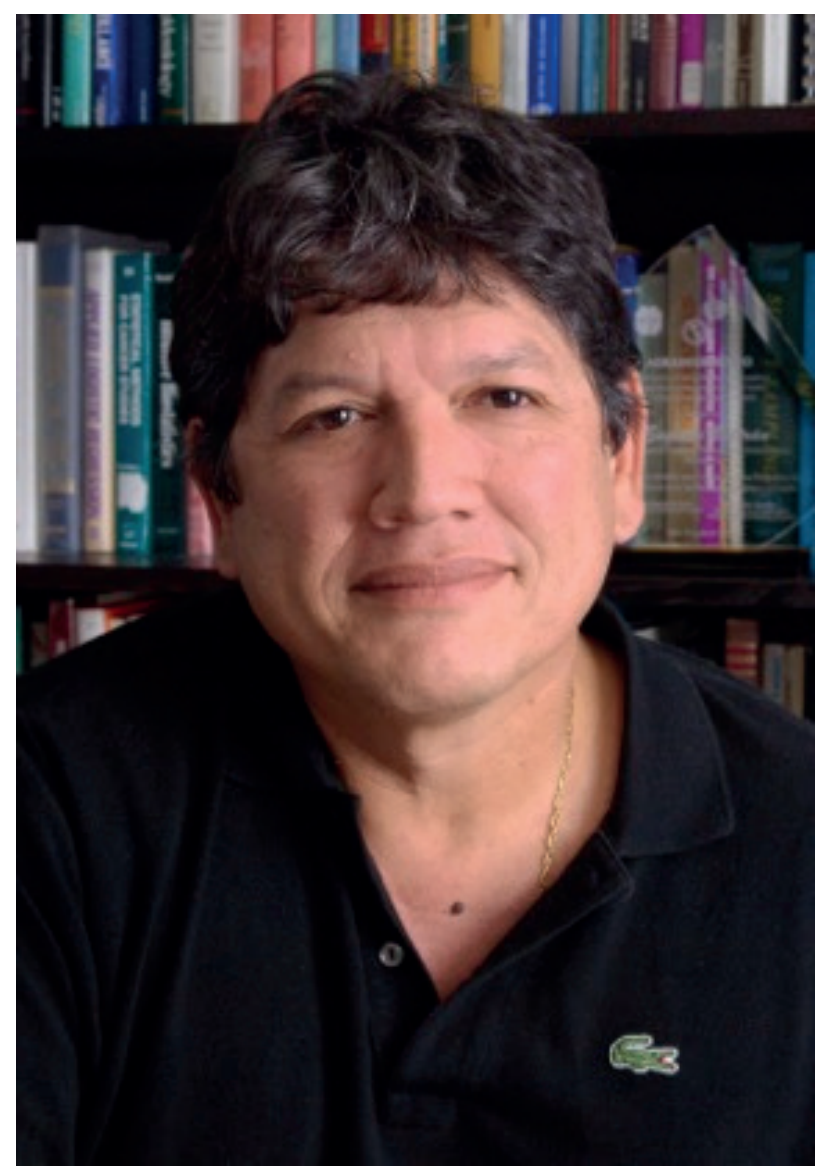

Dr. Eugenio Beltrán-Aguilar 
